\title{
Evaluation of Edge Electron Temperature Fluctuations Using a Conditional Technique on TST-2
}

\author{
Jun'ichi OZAKI, Masateru SONEHARA, Yoshihiko NAGASHIMA ${ }^{1)}$, Yuichi TAKASE ${ }^{1)}$, \\ Akira EJIRI $^{1)}$, Kotaro YAMADA ${ }^{1)}$, Hidetoshi KAKUDA ${ }^{2)}$, Shigeru INAGAKI ${ }^{3)}$, \\ Takuya OOSAKO ${ }^{4)}$, Byung Il AN ${ }^{2)}$, Hiroyuki HAYASHI ${ }^{1)}$, Kentaro HANASHIMA ${ }^{1)}$, \\ Junichi HIRATSUKA ${ }^{2)}$, Hiroaki KOBAYASHI ${ }^{1)}$, Hiroki KURASHINA ${ }^{1)}$, Takuya SAKAMOTO ${ }^{1)}$, \\ Takashi YAMAGUCHI ${ }^{1)}$, Osamu WATANABE ${ }^{1)}$ and Takuma WAKATSUKI ${ }^{2)}$ \\ Faculty of Science, The University of Tokyo, Tokyo 113-0033, Japan \\ ${ }^{1)}$ Graduate School of Frontier Sciences, The University of Tokyo, Kashiwa 277-8561, Japan \\ ${ }^{2)}$ Graduate School of Science, The University of Tokyo, Tokyo 113-0033, Japan \\ ${ }^{3)}$ Research Institute for Applied Mechanics, Kyushu University, Kasuga 816-8580, Japan \\ ${ }^{4)}$ CEA, IRFM, F-13108 Saint-Paul-lez-Durance, France
}

(Received 25 February 2010 / Accepted 1 May 2010)

\begin{abstract}
The amplitudes of electron temperature fluctuations are evaluated in the edge plasma of TST-2 using a new technique. Langmuir probe current-voltage characteristic curves are conditionally reconstituted in terms of the magnitude of the floating potential. High/low electron temperatures are obtained in low/high floatingpotential phases. The relationship between the electron temperature fluctuations and the time-averaged electron temperature gradient is discussed.
\end{abstract}

(C) 2010 The Japan Society of Plasma Science and Nuclear Fusion Research

Keywords: electron temperature fluctuation, edge plasma, spherical tokamak, Langmuir probe

DOI: $10.1585 /$ pfr.5.023

The development of a fast, precise, and highresolution measurement of the electron temperature in edge plasmas is important in fusion plasma research [1,2]. Recent studies on momentum transport by edge turbulence were performed with Langmuir probe (LP) floatingpotential data under the assumption that the electron temperature fluctuation is negligible [3]. However, in highbeta toroidal plasmas (e.g., spherical tori), this assumption may not be valid, and may lead to an incorrect estimation of turbulence transport. In this study, we propose a new approach [4] to estimate the time-averaged and fluctuating components of the electron temperature in turbulent plasmas. This approach focuses on the use of a single-LP measurement to obtain high spatial resolution, instead of the commonly used triple probe technique. First, we describe the experimental setup on TST-2 [5] and the method of conditional classification. Second, we provide an example of data analysis, and estimate the time-averaged and the fluctuating components of the electron temperature. Third, to check the validity of this analysis technique, we form a relationship between the fluctuation amplitude and the time-averaged gradient. Finally, we discuss the results and summarize the study.

Experiments were conducted on the TST-2, which is a spherical tokamak device with major radius $R_{0} \sim 0.38 \mathrm{~m}$, minor radius $a \sim 0.25 \mathrm{~m}$ (aspect ratio $A \geq 1.5$ ), toroidal

author'se-mail: ozaki@fusion.k.u-tokyo.ac.jp magnetic field $B_{\mathrm{t}} \leq 0.3 \mathrm{~T}$, and typical plasma current $I_{\mathrm{p}} \leq 200 \mathrm{kA}$. A multichannel LP was used to measure the floating potential $V_{\mathrm{f}}$, probe voltage $V_{\text {probe }}$, and probe current $I_{\text {probe }}$ simultaneously at a specific point in the edge plasma. The LP is radially movable, and measurements were performed at radial locations of $x=-30,-25,-15$, 0 , and $15 \mathrm{~mm}$, where $x$ is the radial position relative to the low-field side limiter, located at $R=630 \mathrm{~mm}$ ( $R$ is the major radius). The positive $x$ direction is taken outward, away from the plasma center. A slowly varying $(1 \mathrm{kHz})$ bias voltage $V_{\text {probe }}$, from -250 to $50 \mathrm{~V}$, was applied to one electrode of the LP and $I_{\text {probe }}$ was measured on the same electrode. Two other electrodes were used to measure $V_{\mathrm{f}}$. We selected data within the range $22.5-25.5 \mathrm{~ms}$ out of the plasma duration of $16-33 \mathrm{~ms}$, because the plasma is nearly stationary during this interval. Figure 1 (a) shows the waveforms of $V_{\mathrm{f}}$ and $I_{\text {probe }}$ during a single voltage sweep.

We introduce a new conditional classification method. We assume a one-to-one correspondence between $V_{\mathrm{f}}$ and the electron temperature $T_{\mathrm{e}}$. The validity of this assumption is discussed later. This assumption enables us to calculate $T_{\mathrm{e}}$ as a function of $V_{\mathrm{f}}$, and helps estimate the fluctuation of $T_{\mathrm{e}}$ from the fluctuation of $V_{\mathrm{f}}$. Firstly, we categorize the data by $V_{\mathrm{f}}$ at any fixed $x$. Secondly, we estimate $T_{\mathrm{e}}$ and its temporal fluctuation, and derive the dependence of $T_{\mathrm{e}}$ on $V_{\mathrm{f}}$. Thirdly, we calculate the average $T_{\mathrm{e}}$, and the fluctuation of $T_{\mathrm{e}}$ from the fluctuation of $V_{\mathrm{f}}$ at fixed $x$. Finally, 

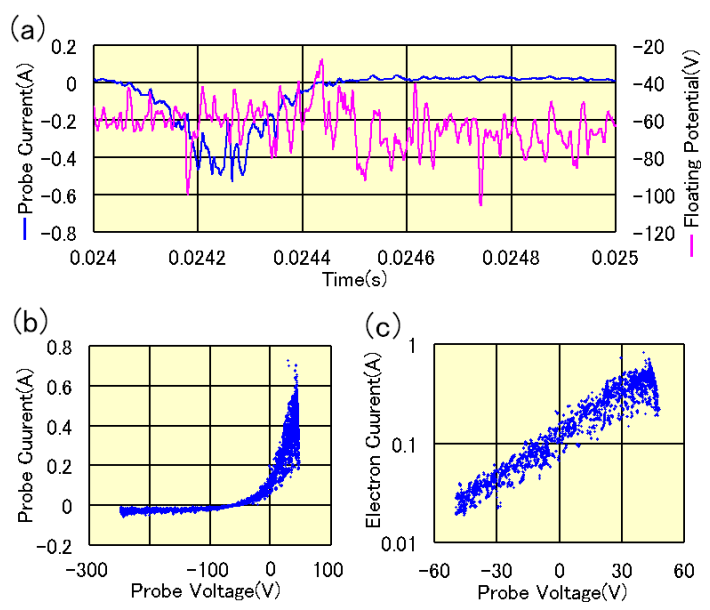

Fig. 1 (a) Waveforms of $V_{\mathrm{f}}$ and $I_{\text {probe }}$ during a single voltage sweep, (b) $V_{\text {probe }}$ and $I_{\text {probe }}$ at $x=-25 \mathrm{~mm}$ and $-76.3 \mathrm{~V}<$ $V_{\mathrm{f}}<-74.9 \mathrm{~V}$, and (c) $V_{\text {probe }}$ and electron current $I_{\text {probe }}-$ $I_{\mathrm{i}, \text { sat }}$ under the same condition.

at any $x$, we estimate the position fluctuations about $V_{\mathrm{f}}$ and $T_{\mathrm{e}}$ from the plot of the average $V_{\mathrm{f}}$ and $T_{\mathrm{e}}$, and from the fluctuations versus $x$ (the average and fluctuation of $V_{\mathrm{f}}$ is directly calculated from the original data).

We categorize the data according to the value of $V_{\mathrm{f}}$, and estimate $T_{\mathrm{e}}$ in each range of $V_{\mathrm{f}}$. Figures $1(\mathrm{~b})$ and (c) show the relationship between $V_{\text {probe }}$ and $I_{\text {probe }}$ in the range $-76.3 \mathrm{~V}<V_{\mathrm{f}}<-74.9 \mathrm{~V}$ measured at $x=-25 \mathrm{~mm}$. In Fig. 1 (b), $I_{\text {probe }}$ becomes nearly constant for $V_{\text {probe }}<$ $-150 \mathrm{~V}$, corresponding to the ion saturation current $I_{\mathrm{i}, \text { sat }}$. The curve is nearly exponential for $V_{\text {probe }}>0 \mathrm{~V}$. We calculate $T_{\mathrm{e}}$ from the semi-log plot of $V_{\text {probe }}$ and electron current $I_{\text {probe }}-I_{\mathrm{i}, \text { sat }}$, such as that shown in Fig. 1 (c).

Now, we illustrate the relationship between $T_{\mathrm{e}}$ and $V_{\mathrm{f}}$. Figure 2 (a) is a plot of average $T_{\mathrm{e}}$ at each $V_{\mathrm{f}}$, taken at $x=$ $-25 \mathrm{~mm}$. Here, we can estimate $\delta T_{\mathrm{e}}$, the fluctuation of $T_{\mathrm{e}}$ caused by $\delta V_{\mathrm{f}}$ (the fluctuation of $V_{\mathrm{f}}$ ). We also evaluate the $T_{\mathrm{e}}$ fluctuation at fixed $V_{\mathrm{f}}$, assuming that the fluctuation of $I_{\text {probe }}$ at fixed $V_{\mathrm{f}}$ originates from the temporal fluctuation of $T_{\mathrm{e}}$. It is of the order $1-2 \mathrm{eV}$, and this is discussed later.

Next, we calculate the fluctuation in position from $V_{\mathrm{f}}$ and $T_{\mathrm{e}}$ data at any $x$. Figure 2 (b) shows the average $V_{\mathrm{f}}$ and average $T_{\mathrm{e}}$, as well as $\delta V_{\mathrm{f}}$ and $\delta T_{\mathrm{e}}$. It is natural that the position fluctuation of $T_{\mathrm{e}}$ at $x, \delta x_{T_{\mathrm{e}}}(x)$, is defined as the distance between $x$ and $x_{\mathrm{f}}$, where $T_{\mathrm{e}}\left(x_{\mathrm{f}}\right)$ is equal to $T_{\mathrm{e}}(x) \pm$ $\delta T_{\mathrm{e}}(x)$ (when there are two $x_{\mathrm{f}}$ 's, we take their average). We define $\delta x_{V_{\mathrm{f}}}(x)$ in the same manner. Figure 2 (c) plots $\delta x_{T_{\mathrm{e}}}(x)$ and $\delta x_{V_{\mathrm{f}}}(x)$.

There are two types of fluctuations for $T_{\mathrm{e}}$ : the fluctuation when $V_{\mathrm{f}}$ is fixed and the fluctuation caused by $\delta V_{\mathrm{f}}$. If the ratio of the former to the latter were small, the assumption of one-to-one correspondence between $V_{\mathrm{f}}$ and $T_{\mathrm{e}}$ is valid, because if this ratio is nearly zero, $T_{\mathrm{e}}$ can be expressed as a function of $V_{\mathrm{f}}$. Thus, this ratio represents the validity of the assumption. In fact, the ratio is about 0.5 . This small ratio indicates that $T_{\mathrm{e}}$ mainly depends on simultaneous $V_{\mathrm{f}}$, and supports our assumption.
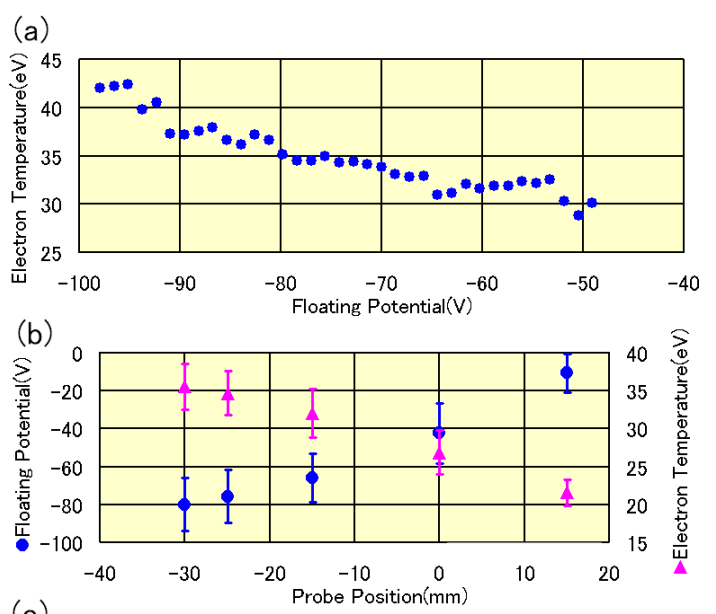

(c)

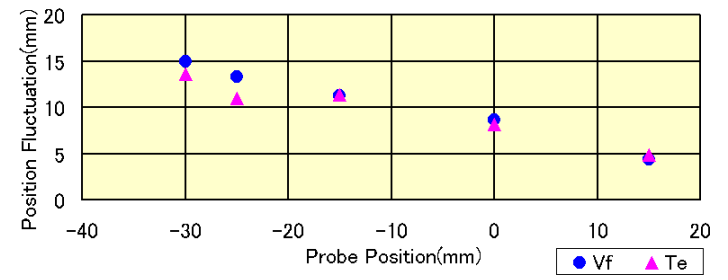

Fig. 2 (a) $T_{\mathrm{e}}$ versus $V_{\mathrm{f}}$ at $x=-25 \mathrm{~mm}$. It is estimated in the range $V_{\text {probe }}>-50 \mathrm{~V}$, (b) average $V_{\mathrm{f}}$ and $T_{\mathrm{e}}$ versus $x$, and $\delta V_{\mathrm{f}}$ and $\delta T_{\mathrm{e}}$ (shown by error bars), and (c) spatial fluctuations of $V_{\mathrm{f}}$ and $T_{\mathrm{e}}$ versus $x$.

Now, we assume a radial fluctuation of velocity from a magnetohydrodynamics-type oscillation, and that the fluctuations of parameters are caused entirely by the oscillation of the plasma with the spatial gradients. Under this assumption, we compare the spatial fluctuations of two scalar parameters, $V_{\mathrm{f}}$ and $T_{\mathrm{e}}$. Figure 2 (c) shows $\delta x_{V_{\mathrm{f}}} \sim \delta x_{T_{\mathrm{e}}}$, which implies $V_{\mathrm{f}}$ and $T_{\mathrm{e}}$ have similar types of fluctuations; both fluctuations have the same origin, the spatial oscillation of the plasma.

In summary, using the new conditional classification technique, we have evaluated the variance of the electron temperature in the edge plasma of the TST-2. The normalized fluctuation level of the electron temperature is around $18 \%$ or higher for the case studied. We compared the relationships between fluctuations and time-averaged gradients in two parameters, and the validity of the analysis was discussed. This study was supported by Japan Society for the Promotion of Science (JSPS) Grants-in-Aid for Scientific Research (S) No. 21226021 and for Scientific Research (A) No. 21246137, and partially by JSPS Grant-inAid for Scientific Research (S) No. 21224014.

[1] J. Boedo et al., Rev. Sci. Instrum. 70, 2997 (1999).

[2] D. Rudakov et al., Rev. Sci. Instrum. 75, 4334 (2004).

[3] G. Tynan et al., Plasma Phys. Control. Fusion 51, 113001 (2009).

[4] S. Inagaki et al., In Proceedings of 24th Annual meetings of Japan Society of Plasma Science and Nuclear Fusion Research, Egret Himeji, Hyogo, Japan, 2007, 28aB14P (in Japanese).

[5] Y. Takase et al., Nucl. Fusion 41, 1543 (2001). 